\title{
Memorias eclipsadas. Duelo y resiliencia comunitaria en la prisión política Jorge Montealegre Iturra
}

Editorial Asterión, Santiago, 2013, 230 págs.

\author{
Geoffrey Durand \\ Paris III Sorbonne Nouvelle, Institut des Hautes Etudes de lAmérique Latine, Paris, France. \\ Email: geoffrey-durand@live.fr \\ “Je n’écrirai pas de poème d'acquiescement” \\ (René Char, Feuillets dHypnos)
}

Alors que la mort mit le point final à la vie de Jorge Semprun au mois de juin 2011, cest à titre posthume que fut publiée sa dernière uvre, Exercices de survie. Ultime introspection dune mémoire qui nous emmène une dernière fois dans la Résistance française, la clandestinité communiste en Espagne et surtout au camp de concentration de Buchenwald, en abordant “autant par lexpérience vécue que par la réflexion” la torture et les capacités humaines de résistance face à la douleur et la désubjectivation. Après un grand voyage, comme Semprun qualifiait sa déportation, "lère du témoin" (Wieviorka, 1998) a montré que pour différentes raisons et répondant à diverses logiques se taire est impossible. Le récent essai de lécrivain et journaliste chilien Jorge Montealegre sinscrit dans la continuation des considérations personnelles émanant des victimes et des travaux académiques sur les expériences-limites en prenant le cas des dictatures militaires des années 1970 dans le Cône Sud latino-américain.

Ce sont presque quarante ans qui se sont écoulés entre le « diplôme dhonneur » que lauteur reçut au sein du camp de Chacabuco de la part des autres prisonniers politiques lors dun concours de poésie et le Prix du Conseil National du livre et de Lecture attribué à son essai, Memorias eclipsadas, duelo y resiliencia comunitaria en la prisión política. En effet, comme le souligne justement Sergio González Rodríguez dans le prologue, Jorge Montealegre se trouve dans une double position en étant à la fois auteur de lessai et ancien prisonnier politique de la Junte militaire qui a renversé le gouvernement de lUnité Populaire par le coup dEtat du 11 septembre 1973. Ce qui nest pas sans rappeler que les premières et rares dans le cas latinoaméricain études portant sur les camps et centres de torture ont été menées par danciens détenus (Langbein 1981; Montealegre 2003; Calveiro 2004; Salazar 2013). Ayant pour origine sa thèse doctorale soutenue à lUniversité 
de Santiago du Chili, lessai de Jorge Montealegre a été remanié afin dêtre accessible à un large public. Le travail final est particulièrement réussi et nous rejoignons la critique du prologue qui le qualifie de "remarquable par son objectivité" et sa "capacité de montrer cette réalité avec la proximité nécessaire et la distance prudente” (p.18).

En sappuyant principalement sur le camp de prisonniers politiques de Chacabuco au Chili et la prison pour femmes "Punta de Rieles" en Uruguay, lauteur mène en cinq chapitres une réflexion globale sur la violence et la résistance collective au cur de ce quil nomme de manière abstraite la "prison politique”. Ainsi, tout en pensant "sur soi-même pour pouvoir penser laltérité" -ce quil fait à travers une approche genrée et transnationale-, lauteur souhaite "établir, théoriquement, les piliers de la résilience communautaire" et donner une valeur à la "mémoire assombrie et discréditée pour reconstruire des expériences qui ont été maintenues au second plan" (p.23). Il ne sagit pas de là "où la nuit na nul besoin détoiles, nulle part on sintéresse à nous" comme lécrivait fatalement Paul Celan, au contraire, les "mémoires éclipsées" sont, selon lauteur, toutes celles relatives à un "bonheur paradoxal” (felicidad paradójica). Ce dernier, vécu dans lenfermement répressif, est incarné par la camaraderie solidaire et les "expressions ludiques, humoristiques et artistiques" déployées par les détenus face au système de déshumanisation et danéantissement physique et psychique.

Si lhistoire culturelle, tout comme lensemble des sciences de lHomme, est restée en retrait de ces problématiques ${ }^{1}$, signalons tout dabord que, fidèle à toute histoire du temps présent, nous sommes face à un "passé qui ne passe pas", ou "trop vite passé" (Compagnon et Gaudichaud, 2008). De plus, la "culpabilité de vivre des survivants" et le besoin de produire un matériau qui servirait avant tout à la condamnation politique et judiciaire des dictatures du Cône Sud ont inhibé "les possibilités de partager des expériences positives" permettant de "surpasser ladversité" (p.24). Enfin, il est important de mentionner que si les fins de lessai sont certes réflexives, les moyens dy parvenir sappuient sur les archives relevant à la fois du domaine privé (lettres de prisonniers, poèmes, dessins, artisanat, témoignages) et public (entretiens, règles pénitentiaires, etc.).

Intitulée "Lennemi interne”, la première partie se consacre à la présentation de ce que furent le camp chilien de Chacabuco et la prison politique uruguayenne "Punta de Rieles" tout en proposant une contextualisation claire et pertinente du tournant des années 1970 dans le Cône Sud. Si lidée directrice de louvrage est de rendre compte des similitudes du renversement de la démocratie par les juntes militaires (de "lextirpation du cancer marxiste » du corps de la nation, de la «transformation morale » de la Patrie, des pratiques de répression, denfermement et délimination physique et mentale de ceux qui deviennent alors "les ennemis internes") et des formes de résistance humaine face à lexpérience-limite, Jorge Montealegre ne peut omettre que les cas sur lesquels il sappuie font figure de deux archipels répressifs différents. Dun côté, Chacabuco (1973-1975) est un camp de prisonniers politiques où il 
ny a que des hommes, occupant les baraques des ouvriers de lancienne mine de salpêtre, soumis à lisolement géographique du désert dAtacama et symbolique du fil de fer barbelé. Au sein du camp, les prisonniers politiques peuvent se déplacer, sorganiser, en négociant avec les gardiens, ce qui avantage une résilience communautaire. A contrario, "Punta de Rieles" (1973-1985), à quinze kilomètres de Montevideo, est juridiquement une prison politique, destinée aux femmes, où lisolement est individuel puisque le bâtiment de trois étages était un ancien noviciat jésuite constitué de cellules. Il ny a aucun dialogue avec les gardiens et la surveillance de chaque détenue est oppressive. Jorge Montealegre propose alors un contexte général destiné à la compréhension du lecteur, rappelant avec justesse les principales caractéristiques de la mise en place des dictatures militaires du Cône Sud. La polarisation du monde au cours de la Guerre froide avec, à léchelle continentale, lenseignement de la Doctrine de Sécurité Nationale, les coups dEtat du Brésil (1964) à lArgentine (1976), le Plan Z et le Plan Condor, etc. Lauteur montre enfin comment "lennemi interne” est-il à la fois fantasmé et éliminé physiquement, culturellement et symboliquement, des autodafés de livres politiques et "subversifs" à la Morale et lOrdre en passant par les “opérations de nettoyage ${ }^{2}$ ” des murs de la ville et des imaginaires de "lidentité marxiste” (cheveux et “bigote Debray” à couper, par exemple).

Le deuxième chapitre, "Situation de deuil”, aborde dune part le processus darrestation et séquestration arbitraires des "ennemis internes" avec le passage par les centres secrets de torture, et dautre part les formes de résilience communautaire. Puisque "on ne peut dire la prison sans parler de son antichambre, la torture” (Trías, 2003), Jorge Montealegre invite le lecteur à une réflexion sur ses mécanismes, la « fugue mentale » (p.75) des victimes et limpact de son traumatisme (“quiebre biográfico”). La faute et la honte, responsables de la désubjectivation et lanéantissement moral, deviennent lennemi intime de la victime qui “débute le chemin de récupération” et voit "le commencement du processus de résilience comparable à la convalescence” (p.81) dès son transfert dans un centre de détention, une prison ou un camp de prisonniers. Lauteur propose également un répertoire des formes de violence, ce qui, malgré la perspective intéressante de genre, manque de nuance en oubliant quil y a aussi, et surtout, une classification entre les divers types de dispositifs denfermement. Enfin, la deuxième partie du chapitre se consacre à la résilience, entendue comme "la capacité de protéger lintégrité même () et de forger un comportement vital positif malgré les circonstances difficiles” (Poletti et Dobbs, 2005). A la célèbre interrogation de Friedrich Hölderlin "à quoi bon des poètes en temps de détresse ?”, Jorge Montealegre renverse la question en montrant le rôle à la fois individuel et collectif de la poésie, parfois faite par des prisonniers qui navaient jamais eu lidée décrire. La parole, plus globalement, devient "cette sortie secrète du labyrinthe" (p.98). Jorge Montealegre ne manque pas de faire référence, parmi les moyens de résistance, ou les “vertus quotidiennes” selon lauteur de “Face à lExtrême” (Todorov, 1991), la dignité, lactivité de lesprit et lattention portée aux autres prisonniers (autocuidado), en particulier aux plus âgés, et la "valeur thérapeutique" (p.103) de la solidarité puisque, au sein de la « prison politique », la dépression des uns 
peut rapidement faire lagonie de tous. Sans oublier les comportements individuels dauto-répression et de lâcheté de certains prisonniers, lauteur esquisse alors une lecture assez complète de la "résilience communautaire” malgré le fait que la prison "Punta de Rieles" nait pas été traitée sous cet angle.

Dans un troisième temps, “Imaginaires, mémoires et vie quotidienne”, lauteur entend faire une sorte danatomie des témoignages des prisonniers politiques et une analyse sur les mécanismes de la mémoire, objet détude désormais convoité mais qui vit toujours une relation problématique avec lhistoire. En reprenant les travaux classiques de Maurice Halbwachs, de Michael Pollak et de Peter Burke, entre autres, Jorge Montealegre souligne limportance de la mémoire sociale, culturelle et la construction du témoignage, notre principale source pour simmiscer dans "la nouvelle quotidienneté” des prisonniers (p.122). Lauteur décompose avec beaucoup de pertinence et dobjectivité linfluence du "Mal absolu nazi” incarné par les imaginaires de la Shoah ${ }^{3}$, devenu "une figure rhétorique” (p.125) dénonciatrice des régimes militaires du Cône Sud, en soulignant justement les dangers dun tel rapprochement que lon retrouve encore aujourdhui dans certains témoignages et productions scientifiques. Puis, le lecteur trouvera dans la seconde partie de ce chapitre une étude des objets et lieux de mémoire, mêlant intimement aussi bien lartisanat confectionné dans la "prison politique" que les souvenirs, visibles ou non, "marques physiques qui peuvent se manifester par une cicatrice, un tatouage () qui resteront pour toujours témoignant de la torture ou dune situation traumatique” (p.141).

La quatrième partie du livre, "Demandes et revendications de mémoires”, questionne les enjeux qui occupent habituellement la problématique mémorielle pour lhistorien du temps présent. Nous retrouvons un panel des divers types de mémoires, leur (sous-)représentation, les acteurs (le juge, le politique, lhistorien, le journaliste, etc.) qui les conditionnent, le devoir de mémoire” (p.159) et le “droit à loubli” (p.184). Lauteur présente également une analyse primordiale visant à contextualiser et problématiser le rôle du témoignage et de larchive orale en retraçant les différents motifs de cette production littéraire et mémorielle et ses défis pour lhistorien (des “illusions de la mémoire”, p.177, au faux témoignage dEduardo Labarca, Una vida por la legalidad, p.179).

Enfin, le chapitre qui conclut le livre récapitule le résultat de son essai dédié à la confrontation entre le “deuil et la résilience communautaire”, présenté dans une perspective plus globale de réflexions sur la mémoire et la place du témoignage, leurs enjeux pour lhistoire du temps présent et le sens dune histoire " au ras du sol » de la "prison politique". Après avoir appelé au “devoir de réflexion” (p.188), lauteur justifie limportance de la perspective genrée de létude de lexpérience traumatique et denfermement dans la mesure où la voix des femmes, victimes des dictatures, auraient été oubliée par rapport à une mémoire de militants politiques et révolutionnaires (Sapriza, 2009). Au cur du sujet, la résistance individuelle et surtout 
collective à la torture, à la prison ou au camp de prisonniers politiques repose sur trois piliers. Premièrement, ce que lauteur nomme la "reconnaissance du patrimoine socioculturel partagé" (reconocimiento del acervo sociocultural compartido, p.206) qui regroupe les conditions nécessaires au respect et à la confiance du collectif de prisonniers politiques, telles la solidarité, une estime et une attention positives pour les autres. Deuxièmement, le développement dexpressions ludiques et humoristiques dans le contexte de lenfermement et du "deuil”. Troisièmement, la valorisation et la manifestation de la créativité, artisanale ou artistique, permettant le dépassement de soi, lappartenance collective et la production de sens au centre du non-lieu.

Ainsi, nous saluons louvrage de Jorge Montealegre qui, en adaptant des problématiques posées par et pour dautres évènements traumatiques de "lAge des Extrêmes" en Europe, a contribué avec rigueur et clarté à létude dun champ encore trop peu investi par lensemble des sciences humaines et sociales dans le cas de lAmérique latine. Toutefois, nous pourrions regretter lutilisation maladroite et trop générique du terme "prison politique" pour désigner un large éventail de lieux punitifs et disolement des "indésirables" -lenjeu dun tel sujet étant aussi sémantique-, ainsi que le choix danalyser parfois que partiellement - le camp de Chacabuco au Chili et la prison de "Punta de Rieles" en Uruguay qui peut paraitre opportun. Lauteur, parti à son tour à la recherche de "la région cruciale de lâme, où le Mal absolu soppose à la fraternité” (Malraux 1976 : 838), nous propose cet essai comme une invitation qui nous conduirait à une histoire culturelle du quotidien et de la résistance dans lensemble des systèmes répressifs mis en place par les dictatures militaires du Cône Sud ce qui, à plus forte raison, serait une nouvelle voie pour éclaircir les années sombres du continent.

"Puis voilà que tout à coup je commençai à mapercevoir dans une brume, au fond du miroir, dans une brume comme à travers une nappe deau; et il me semblait que cette eau glissait de gauche à droite, lentement, rendant plus précise mon image, de seconde en seconde. Cétait comme la fin dune éclipse” (Maupassant, Le Horla, 1887) 
Polis, Revista Latinoamericana, Volumen 12, $N^{\circ}$ 36, 2013

\section{Notas}

${ }^{1}$ Nous faisons référence à Pradenas Chuecas Luis, « Le théâtre et lunivers concentrationnaire. Représentation de lexpérience dans les camps de concentration au Chili ", in Univers répressifs. Péninsule ibérique et Amérique latine, Paris, LHarmattan, 2001; Joubert-Anghel Valérie, «Des prisons de la dictature aux représentations de lenfermement sur les murs du Chili », in Tauzin-Castellanos (coord.) Prison dAmérique latine: du réel à la métaphore de lenfermement, Bordeaux, Presses Universitaires de Bordeaux, 2009.

${ }^{2}$ A ce sujet, voir: Leiva Quijada Gonzalo et Errázuriz Luis Hernán, El golpe estético. Dictadura militar en Chile (1973-1989), Santiago, Ocho Libros, 2012.

${ }^{3}$ Nous pouvons renvoyer aux ouvrages de Kertesz Imre, LHolocauste comme culture, Arles, Actes Sud, 2009 et Baer Alejandro, Holocausto: recuerdo y representación, Madrid, Losada, 2006. 